\title{
Auditory Cortical Neurons Convey Maximal Stimulus-Specific Information at Their Best Frequency
}

\author{
Nathan Montgomery and Michael Wehr \\ Institute of Neuroscience and Department of Psychology, University of Oregon, Eugene, Oregon 97403
}

\begin{abstract}
Sensory neurons are often thought to encode information about their preferred stimuli. It has also been proposed that neurons convey the most information about stimuli in the flanks of their tuning curves, where firing rate changes most steeply. Here we demonstrate that the responses of rat auditory cortical neurons convey maximal stimulus-specific information about sound frequency at their best frequency, rather than in the flanks of their tuning curves. Theoretical work has shown that stimulus-specific information shifts from tuning curve slope to peak as neuronal variability increases. These results therefore suggest that with respect to the most informative regions of the tuning curve, auditory cortical neurons operate in a regime of high variability.
\end{abstract}

\section{Introduction}

Sensory neurons are often thought of as feature detectors, whose firing rates signal how close a stimulus is to the preferred stimulus of the neuron. For example, neurons in the auditory cortex are tuned for sound frequency, such that they have a best frequency which evokes the maximal firing rate. If neurons are viewed as feature detectors, one might intuitively expect them to convey maximal information about sound frequency for the stimuli closest to their best frequency. However, the flanks of the tuning curve, which have the steepest slope, also provide information about the stimulus. Indeed, it is in this region of the tuning curve that small changes in sound frequency produce large changes in the firing rate. Because the slope of the tuning curve is flat near its peak, responses to neighboring frequencies will be similar, and neurons may therefore be less able to discriminate frequencies near their best frequency than frequencies in the flanks. Thus these two intuitive views of neuronal tuning make quite different predictions about the most informative region of the receptive field.

Recently these two viewpoints were reconciled in an elegant theoretical study, which used a new measure called stimulusspecific information to demonstrate that both interpretations can be correct depending on neuronal variability (Butts and Goldman, 2006). When trial-to-trial variability is high, a model neuron conveys maximal stimulus-specific information at the peak of its tuning curve (Fig. 1a). As trial-to-trial variability is reduced, the maximal information gradually shifts to the steep flanks of the tuning curve (Fig. $1 b, c$ ). This unified framework suggests that neural coding strategies depend critically on the level of neuronal variability.

Received May 6, 2010; revised Aug. 10, 2010; accepted Aug. 15, 2010.

We thank Xiang Gao for assistance with data collection, and Terry Takahashi, Bill Roberts, Kip Keller, Dave Rowland, and Shawn Lockery for helpful discussions.

Correspondence should be addressed to Michael Wehr, 1254 University of Oregon, Eugene, OR 97403. E-mail: wehr@uoregon.edu.

DOI:10.1523/JNEUROSCI.2899-10.2010

Copyright $\odot 2010$ the authors $\quad 0270-6474 / 10 / 3013362-05 \$ 15.00 / 0$
Which regions of the tuning curve convey maximal information in real neurons? Butts and Goldman (2006) based their conclusions on synthetic data generated by model neurons with parameters fit to published recordings. Here we addressed this question directly by measuring the stimulus-specific information that auditory cortical neurons convey about sound frequency. We assume that the task of the neuron is to report sound frequency by firing a certain number of spikes. We find that the stimulus-specific information is always maximal at a neuron's best frequency. We never observed information peaks in the flanks of tuning curves. This suggests that despite the high reliability of auditory cortical neurons (DeWeese et al., 2003), they are still too variable to be most informative at the flanks of their tuning curves, and instead act as feature detectors for their best frequency.

\section{Materials and Methods}

Physiology. We recorded from the left primary auditory cortex of anesthetized (30 mg/kg ketamine, $0.24 \mathrm{mg} / \mathrm{kg}$ medetomidine) male and female rats aged $20-50 \mathrm{~d}$ postnatal. All procedures were in strict accordance with the National Institutes of Health guidelines as approved by the University of Oregon Animal Care and Use Committee. Recordings were made from primary auditory cortex (A1) as determined by the frequency-amplitude tuning properties of cells and local field potentials, based on the criteria of Polley et al. (2007). For single-unit recordings (21 cells) we used the loose cell-attached patch recording method (DeWeese et al., 2003) with 3-4 M $\Omega$ pipettes with an internal solution of $0.9 \%$ saline. Subpial depth of cell-attached recordings ranged from 140 to 670 $\mu \mathrm{m}$ (mean: $383 \mu \mathrm{m}$ ), as determined from micromanipulator travel. For multiunit recordings (86 recording sites) we used 1-2 M $\Omega$ tungsten electrodes amplified with an A-M Systems 1800 extracellular amplifier (bandpass filtering $300-5000 \mathrm{~Hz}$ ). Subpial depth of multiunit recordings ranged from 296 to $583 \mu \mathrm{m}$ (mean: $452 \mu \mathrm{m}$ ). Spike trains were extracted by thresholding the extracellular voltage signal at 4 SDs.

Stimuli. To characterize frequency tuning, we used a pseudorandomly interleaved tone array that consisted of either 17,20,22, or 50 frequencies (logarithmically spaced from 1 to $40 \mathrm{kHz}$ ) at either 20, 40, or $60 \mathrm{~dB}$ SPL, with $25 \mathrm{~ms}$ duration, $5 \mathrm{~ms} 10-90 \%$ cosine-squared ramps, and a 350 or $500 \mathrm{~ms}$ interstimulus interval. Note that values of the stimulus-specific information (see below) are not affected by the number or density of 
a

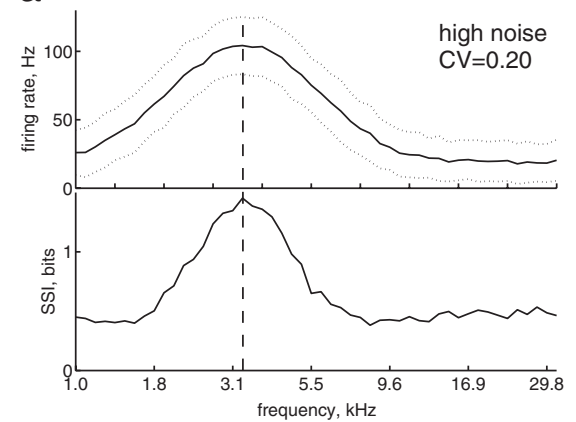

C

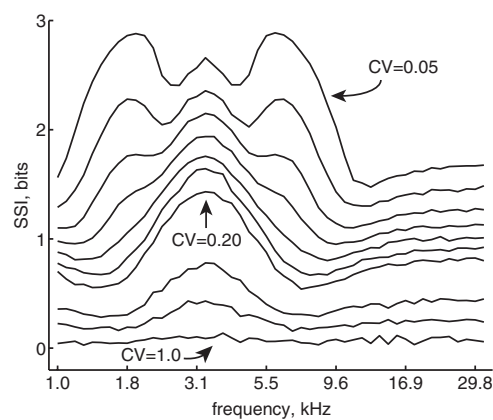

b

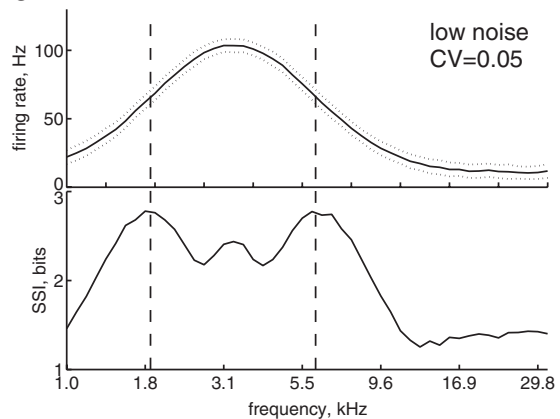

d

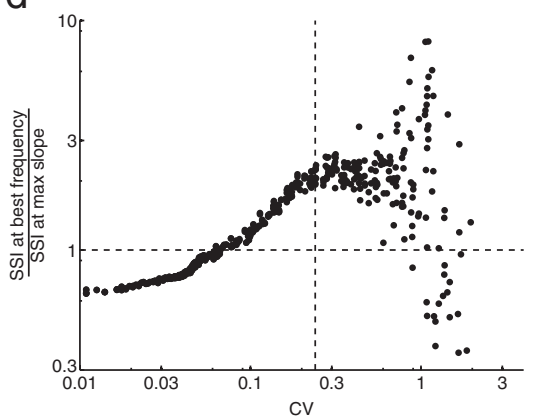

Figure 1. Simulation of the effect of variability on maximal SSI with synthetic data. $\boldsymbol{a}$, Top, Frequency tuning curve of a multiunit recording site in auditory cortex that was used to generate synthetic spike trains with additive Gaussian noise producing high trial-to-trial variability ( $C V=0.20$ at best frequency). Dotted lines indicate one SD. Bottom, SSI as a function of frequency. Maximal SSI was aligned with peak of the tuning curve (vertical dashed line). $\boldsymbol{b}$, Same tuning curve but with low trial-to-trial variability ( $(\mathrm{V}=0.05)$. Maximal SSI has now shifted to the points of steepest slope in the tuning curve (vertical dashed lines). c, SSI curves across a range of variability levels, showing the transition of maximal $\mathrm{SSI}$ from peak to slope of the tuning curve. $\boldsymbol{d}$, The ratio of the SSI at best frequency to SSI at maximum tuning curve slope, as a function of $\mathrm{CV}$ at best frequency. The horizontal dashed line indicates a ratio of 1 (where SSI is equal for best frequency and slope), and the vertical dashed line indicates a CV of 0.24 (the minimum (V in our sample of recordings; see Fig. 3c).

frequencies in the tone array. Number of repetitions was $10-40$ for single-unit and 20 for multiunit recordings. Stimuli were delivered with either a Stax SR-303 speaker or a Pyramid PMBTW43 tweeter, in freefield configuration (speaker located $15 \mathrm{~cm}$ lateral to, and facing, the contralateral ear) in a sound isolation chamber with anechoic surface treatment.

Analysis. We calculated stimulus-specific information (SSI) according to the method of Butts and Goldman (2006) (see also Butts, 2003):

$$
S S I(f)=\sum_{r} p(r \mid f) i_{s p}(r)
$$

where $i_{s p}$ is the specific information (DeWeese and Meister, 1999),

$$
i_{s p}(r)=-\sum_{f} p(f) \log _{2} p(f)+\sum_{f} p(f \mid r) \log _{2} p(f \mid r)
$$

with sound frequency $f$ and response $r$. We quantified spiking responses $r$ as the spike count in a $100 \mathrm{~ms}$ window following tone onset; using 50 or $75 \mathrm{~ms}$ windows did not change the results (data not shown). We did not subtract spontaneous firing rates, which were $16.2 \pm 32.2 \mathrm{~Hz}$ for multiunit recordings and 7.3 $\pm 16.3 \mathrm{~Hz}$ for cell-attached recordings (mean \pm $\mathrm{SD}$ ). We used $p(f)=\frac{1}{S}$ where $S$ is the number of (equiprobable) frequencies in a given tone array. We estimated the SD of the SSI using bootstrap resampling (with 1000 resamples; varying this number over a wide range did not affect the estimate).

Information measures are subject to bias because of the finite number of trials in experiments, which causes undersampling of the relevant probability distributions (for review, see Panzeri et al., 2007). We compared two methods of correction for this bias. The first was to apply the Treves-Panzeri correction to the specific information (Treves and Panzeri, 1995):

$$
i_{s p \text { corrected }}=i_{s p}-C_{1}
$$

where $C_{1}=\frac{(S-1)(R-1)}{2 N \ln 2}, R$ is the number of unique spike counts $r$, and $N$ is the number of experimental tone-response combinations. The second method was to compute the SSI as above, but with all tone-response combinations randomly shuffled. In this case, the response is completely uncorrelated with the stimulus and the true SSI is zero. However, the direct estimate of the SSI produces a positive value, due to bias, and we used this value averaged across stimuli as an estimate of the bias. The corrected SSI is then given by the following:

$$
S S I_{\text {corrected }}=S S I-\left\langle S S I_{\text {shuf }}\right\rangle
$$

These two bias correction methods agreed very closely (Fig. 2a, bottom; compare solid and dashed lines), and we therefore picked one (the shuffle correction) and used it for the remainder of the analysis. Both are probably slight overestimates of the bias, since the corrected information was sometimes slightly negative for frequencies far from best frequency.

We note two further points about bias. First, bias depends on the number of possible neuronal responses, which differs tremendously if one is comparing the information conveyed by spike counts to the information conveyed by temporal codes (as is commonly done). Since we only consider spike count responses, this difference in bias is not a concern. Moreover, the bias for spike counts will be very small compared with that for temporal codes. Second, the bias corrections we used do not vary with tone frequency (i.e., the bias is uniform across the entire tuning curve). Since our goal here is to compare the SSI in different regions of the tuning curve, our main results should be relatively unaffected by bias.

We obtained a tuning curve at each sound level by measuring trialaveraged spike count as a function of sound frequency, and estimated best frequency (at each sound level) as the $\mu$ of a Gaussian fit to this tuning curve. We restricted our analysis to well defined tuning curves according to the following criteria: (1) the goodness of Gaussian fit was required to be $R^{2}>0.9$, and (2) best frequency (as given by $\mu$ of the Gaussian fit) was required to lie within the range of frequencies presented.

Model. To illustrate how decreased trial-to-trial variability can shift the maximal SSI from the peak to the flanks of the tuning curve (Fig. 1), we used a model neuron to generate synthetic data. The responses (spike count on each of 250 trials) of the model neuron were given by a tuning curve with additive Gaussian-distributed noise. The tuning curve we used was from an actual multiunit recording (Fig. $2 a$, the example recording shown).

\section{Results}

We first used a simple model neuron to generate synthetic data to characterize how SSI (see Materials and Methods) shifts from the best frequency to the slope of the tuning curve as a function of trial-to-trial variability. The model consisted of the tuning curve from one of our multiunit recordings from primary auditory cortex, combined with a variable amount of additive Gaussiandistributed noise. When we set the trial-to-trial variability to be relatively high, corresponding to a coefficient of variation $(\mathrm{CV})$ of 
the spike count at best frequency of $\sim 0.2$, SSI was maximal at the peak of the tuning curve (Fig. 1a). At a much lower variability, the SSI at the slopes of the tuning curve became greater than that at the best frequency (Fig. 1b). The gradual shift from maximal SSI at the slopes to maximal SSI at best frequency can be seen in Figure $1 c$. These results agree well with those of Butts and Goldman (2006). To quantify this shift, we took the ratio of the SSI at best frequency to the SSI at the steepest slope of the tuning curve (Fig. $1 d)$. For very low variability (CV $<\sim 0.06$ ), SSI at the slopes was greater than at best frequency, producing a ratio $<1$. At higher variability, SSI was greater at best frequency, producing a ratio $>1$. At even higher variability $(\mathrm{CV}>\sim 0.3)$, the overall amount of SSI drops, and also flattens out as a function of frequency (for example, see the curve for $\mathrm{CV}=1.0 \mathrm{in} \mathrm{Fig.}$ $1 c)$. As a result, the ratio in Figure $1 d$ becomes noisy as the variability surpasses $\mathrm{CV}=1$. These results characterize how the location of peak SSI depends on trialto-trial variability, and in particular show that the transition from slope to best frequency occurs over the $\mathrm{CV}$ range from $\sim 0.05$ to 0.1 .

We next measured the stimulus-specific information that auditory cortical neurons conveyed about sound frequency. Figure $2 a$ (top) shows the frequency tuning curve of a typical multiunit recording site measured at $60 \mathrm{~dB}$ SPL. The best frequency for this tuning curve was $3.3 \mathrm{kHz}$, as given by $\mu$ from the Gaussian fit. The SSI (Fig. $2 a$, bottom) was essentially congruent with the tuning curve, with a peak (at $3.3 \mathrm{kHz}$ ) at the best frequency, and no major peaks on the flanks of the tuning curve. Thus this recording site conveyed maximal information at best frequency rather than at the slopes of the tuning curve.

The example in Figure $2 a$ was typical of our population of single-unit and multiunit recordings. Figure $2 b$ (top) shows the population-average tuning curve across our sample of 197 tuning curves from 21 single-unit and 86 multiunit recordings (tuning curves were collected at more than one sound level for each site). The population-average SSI for these recording sites (Fig. $2 b$, bottom) was maximal at best frequency, and showed no peaks at the slopes of the tuning curve. This was also true for individual recording sites across the population. Figure $3 a$ shows the relationship between the best frequency and the sound frequency at which SSI was maximal, for each tuning curve. Points cluster around the line of unity slope, indicating that SSI is maximal at or near best frequency for individual sites as well as for the populalevels per neuron).
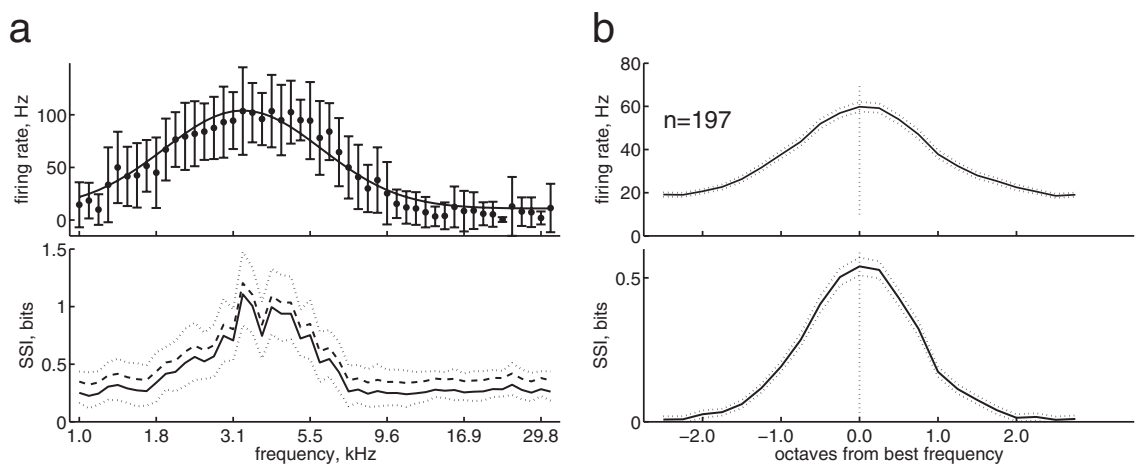

Figure 2. Maximal SSI is aligned to best frequency in auditory cortical neurons. $\boldsymbol{a}$, Top, Frequency tuning curve of a multiunit recording in A1. Dots indicate mean spike counts, error bars indicate SD, and solid line indicates a Gaussian fit. Bottom, SSI for this recording as a function of frequency. Solid line is SSI with Treves-Panzeri bias correction, dashed line is SSI with shuffle bias correction, and dotted lines indicate SD estimated by bootstrap resampling. $\boldsymbol{b}$, Top, Population-average tuning curve, aligned to best frequency for each recording site. Bottom, Population-average SSI. Dotted lines indicate SEM ( $n=197: 164$ tuning curves from 86 multiunit recording sites, and 33 tuning curves from 21 single units; this includes tuning curves collected at multiple sound

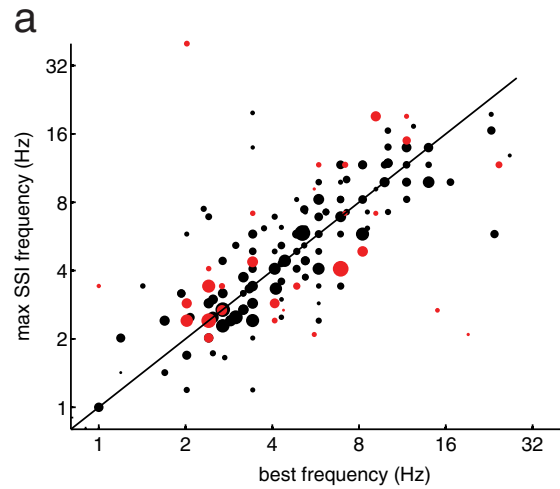

C

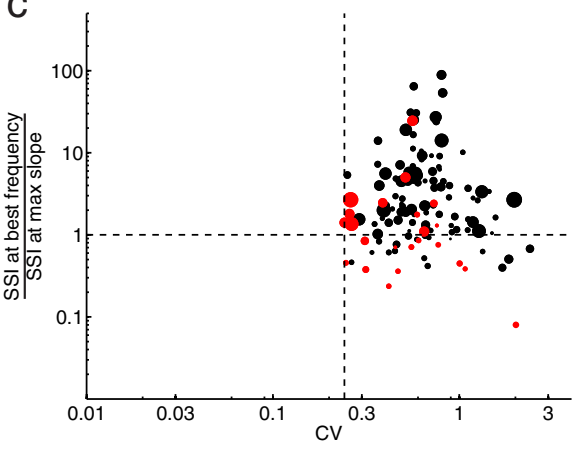

Figure 3. Maximal SSI corresponds to best frequency, not steepest slope. $\boldsymbol{a}$, For each tuning curve, the frequency at which SSI was maximal is plotted against best frequency. Values cluster around the line of unity slope (solid line). In $\boldsymbol{a}-\boldsymbol{c}$, black dots indicate multiunit recordings, red dots indicate single-unit recordings, and dot radius is proportional to maximal SSI. $\boldsymbol{b}$, For each tuning curve, the SSI at best frequency is plotted against the SSI at the two points of steepest slope of the tuning curve (one dot each for the rising and falling slopes). Values were truncated at zero. Values cluster above the line of unity slope (solid line), indicating that the SSI at best frequency is greater than the SSI at the steepest slope. $c$, The ratio of the SSI at best frequency to SSI at maximum tuning curve slope, as a function of $\mathrm{CV}$ at best frequency. The horizontal dashed line indicates a ratio of 1 (corresponding to equal SSI for best frequency and slope), and the vertical dashed line indicates a $\mathrm{CV}$ of 0.24 (the minimum $\mathrm{CV}$ in our sample of recordings; compare with Fig. 1d).

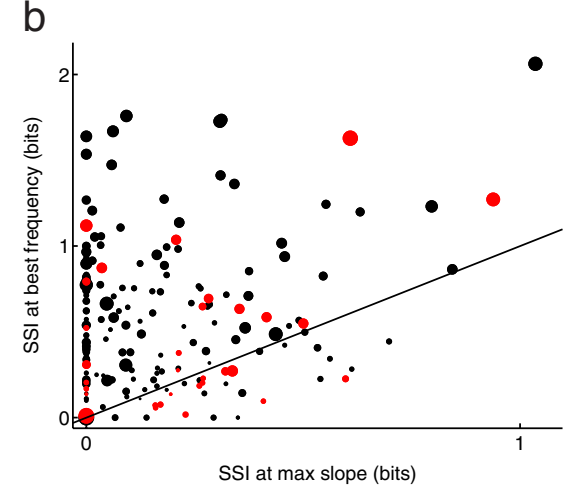

- single-unit

(1.0 
was also true for each sound level computed independently (data not shown).

These results suggest that, as a population and individually, auditory cortical neurons have far too much trial-to-trial variability in spike count to convey maximal SSI at their tuning curve slopes. We wondered whether neurons in our sample with lower variability might show a trend toward shifting information from the best frequency to the slopes of their tuning curves. To characterize the amount of trial-to-trial variability in our recordings and whether this shifts the location of peak SSI, we plotted the ratio of the SSI at best frequency to the SSI at the steepest slope of the tuning curve against the $\mathrm{CV}$ at best frequency, for all tuning curves in our sample (Fig. 3c). This is the same analysis shown for synthetic data in Figure $1 d$. The lowest CV in our sample was 0.24 , which was well above the transition range of 0.05-0.1 that we identified by synthetically manipulating variability (compare with Fig. $1 d$ ). This value of $C V=0.24$ is indicated by the vertical dashed line in Figure $3 c$ and for reference in Figure $1 d$. This suggests that all the neurons in our sample had much greater trial-to-trial variability than would be required to show a peak in the SSI in the flanks of their tuning curves.

As another test of whether neurons in our sample might show a trend toward shifting information from the best frequency to the slopes of their tuning curves depending on their trial-to-trial variability, we performed a median split of our population into recording sites with high and low trial-to-trial variability in spike count. The difference between the SSI at best frequency and at the slope was not significantly different in these two halves of the population (paired one-tailed $t$ test). This suggests that the coding advantage of best frequency over the tuning curve slope is consistent across the population as a whole.

We wondered whether trial-to-trial variability might vary as a function of sound level, and whether this might shift the location of maximal SSI for tuning curves measured at different sound levels. The CV at best frequency decreased slightly but significantly with increasing sound level (linear regression slope: -0.01 $\mathrm{dB}^{-1}, p<10^{-7}$ ), indicating that spike count responses were more reliable for louder tones. However, the ratio of the SSI at best frequency to the SSI at the steepest slope of the tuning curve (same ratio as Fig. 3c) was independent of sound level. Thus even though louder sounds produced more reliable responses, these were still far too variable to produce a shift in the location of maximal SSI in the tuning curves. For even louder sounds (for example, at $80 \mathrm{~dB}$ ), auditory cortical neurons responded over almost the entire audible frequency range, and the SSI therefore decreased overall and flattened out as a function of frequency (data not shown).

How much do these results depend on the specifics of how we measured neuronal responses? To test whether these findings extend beyond spike counts to temporal coding, we reanalyzed our multiunit data using first-spike latency as the response measure. Tuning curves defined by first-spike latency were well tuned and inversely related to spike count tuning curves (supplemental Fig. S1 $a$, available at www.jneurosci.org as supplemental material). The SSI was essentially congruent with the tuning curve, regardless of whether responses were defined by latency or spike count, and independent of response variability (supplemental Fig. S1b, available at www.jneurosci.org as supplemental material). This suggests that our findings are robust and do not critically depend on the response measure, and indeed hold for both temporal and rate codes.

\section{Discussion}

Here we have demonstrated that auditory cortical neurons convey maximal stimulus-specific information about sound frequency at their best frequency, rather than in the flanks of their tuning curves. This finding agrees well with the intuitive notion of neurons as feature detectors, whose spiking conveys information about the presence of their preferred stimulus. In contrast, our results do not support the equally intuitive notion that neurons should best encode differences between stimuli in the high-slope region of their tuning curves. Theoretical work has shown that the maximal SSI shifts from tuning curve slope to peak as neuronal variability increases (Butts and Goldman, 2006). We did not observe such a shift in the maximal SSI, suggesting that all of our auditory cortical neurons had too much trial-to-trial variability to show peak SSI in the slopes of their tuning curves, at least with respect to frequency encoding and in our experimental conditions. These findings did not depend on the response measure, and were comparable whether we used a temporal code or a rate code.

Many studies have reported relatively high trial-to-trial response variability across different sensory cortical areas (Dean, 1981; Softky and Koch, 1993; Kara et al., 2000; Arabzadeh et al., 2004; Carandini, 2004), suggesting that these findings might generalize to other cortical areas. A common benchmark for trial-totrial variability is the Poisson process, in which spikes occur randomly and independently, and which has a variance-to-mean ratio (also called the Fano factor) of 1. Cortical neurons are typically reported to be supra-Poisson (variance-to-mean $>1$ ), and our population data are consistent with this: average varianceto-mean ratio at best frequency was 1.5 for single-unit and 3.2 for multiunit recordings. However, $47 \%$ of our single-unit recordings were sub-Poisson, and the lowest variance-tomean ratio in our sample was 0.22 , consistent with a previous report that a subset of auditory cortical neurons show low variability (DeWeese et al., 2003). However, even these low values of variability are still approximately an order of magnitude higher than that required to shift maximal information to the slopes of the tuning curve (compare Figs. $3 c$ and $1 d$; the shift in the synthetic data occurred at CV $0.05-0.1$ or at variance-to-mean 0.02-0.05).

While frequency tuning is a fundamental aspect of auditory cortical neurons, they can also show tuning to other acoustic parameters (Wang et al., 2005). It is therefore possible that neurons could convey maximal information in the slopes of their tuning curves for acoustic parameters other than sound frequency (McAlpine et al., 2001).

Maximal information in the flanks of a tuning curve indicates that responses are informative for making fine frequency discriminations between similar frequencies. In contrast, maximal information at the peak of a tuning curve indicates that responses are most informative about the coarse discrimination between the best frequency and other, distant frequencies (Butts and Goldman, 2006). Intuitively, coarse discriminations are robust to the presence of high trial-to-trial variability, whereas fine discriminations between firing rates are only possible when trial-totrial variability is low. Rats are capable of fine frequency discriminations, with a discrimination limit of $\sim 3 \%$ (Talwar and Gerstein, 1998), similar to that of other mammals (except humans). Our results that maximal SSI always occurred at best frequency suggest that spike counts of individual cortical neurons could support coarse discriminations, but that fine discriminations would require readout of a population code. 
Are these findings, which were obtained in anesthetized animals, expected to generalize to coding of sound frequency in awake animals as well? Previous studies comparing response variability in A1 of awake and anesthetized rodents suggest that they might. Anesthesia has been reported to either have no effect on response variability (with equithesin; Gaese and Ostwald, 2001) or to decrease trial-to-trial variability (barbiturate-ketamine; Ter-Mikaelian et al., 2007) compared with awake rodents. Similarly, increasing the depth of ketamine anesthesia can actually decrease trial-to-trial variability in rat A1 (Kisley and Gerstein, 1999). If variability is the same or higher in awake animals than under anesthesia, then maximal SSI would be expected to remain at the best frequency, rather than shifting to tuning curve flanks, which would be expected in a lower-variability regime. A direct test of this would require frequency tuning curves in awake animals.

Here we have only considered neurons individually, whereas a population of neurons may be able to operate in a lower-noise regime by averaging out uncorrelated noise (Butts and Goldman, 2006). Because trial-to-trial variability is correlated across neurons (Zohary et al., 1994), a test of this idea would require tuning curves from simultaneously recorded neurons. It would be very interesting to see whether maximal SSI might shift from the peak to the slope of tuning curves when SSI is calculated from increasing numbers of simultaneously recorded auditory cortical neurons.

\section{References}

Arabzadeh E, Panzeri S, Diamond ME (2004) Whisker vibration information carried by rat barrel cortex neurons. J Neurosci 24:6011-6020.

Butts DA (2003) How much information is associated with a particular stimulus? Network 14:177-187.

Butts DA, Goldman MS (2006) Tuning curves, neuronal variability, and sensory coding. PLoS Biol 4:e92.
Carandini M (2004) Amplification of trial-to-trial response variability by neurons in visual cortex. PLoS Biol 2:E264.

Dean AF (1981) The variability of discharge of simple cells in the cat striate cortex. Exp Brain Res 44:437-440.

DeWeese MR, Meister M (1999) How to measure the information gained from one symbol. Network 10:325-340.

DeWeese MR, Wehr M, Zador AM (2003) Binary spiking in auditory cortex. J Neurosci 23:7940-7949.

Gaese BH, Ostwald J (2001) Anesthesia changes frequency tuning of neurons in the rat primary auditory cortex. J Neurophysiol 86:1062-1066.

Kara P, Reinagel P, Reid RC (2000) Low response variability in simultaneously recorded retinal, thalamic, and cortical neurons. Neuron 27:635646.

Kisley MA, Gerstein GL (1999) Trial-to-trial variability and state-dependent modulation of auditory-evoked responses in cortex. J Neurosci 19:1045110460.

McAlpine D, Jiang D, Palmer AR (2001) A neural code for low-frequency sound localization in mammals. Nat Neurosci 4:396-401.

Panzeri S, Senatore R, Montemurro MA, Petersen RS (2007) Correcting for the sampling bias problem in spike train information measures. J Neurophysiol 98:1064-1072.

Polley DB, Read HL, Storace DA, Merzenich MM (2007) Multiparametric auditory receptive field organization across five cortical fields in the albino rat. J Neurophysiol 97:3621-3638.

Softky WR, Koch C (1993) The highly irregular firing of cortical cells is inconsistent with temporal integration of random EPSPs. J Neurosci $13: 334-350$.

Talwar SK, Gerstein GL (1998) Auditory frequency discrimination in the white rat. Hear Res 126:135-150.

Ter-Mikaelian M, Sanes DH, Semple MN (2007) Transformation of temporal properties between auditory midbrain and cortex in the awake Mongolian gerbil. J Neurosci 27:6091-6102.

Treves A, Panzeri S (1995) The upward bias in measures of information derived from limited data samples. Neural Comput 7:399-407.

Wang X, Lu T, Snider RK, Liang L (2005) Sustained firing in auditory cortex evoked by preferred stimuli. Nature 435:341-346.

Zohary E, Shadlen MN, Newsome WT (1994) Correlated neuronal discharge rate and its implications for psychophysical performance. Nature 370:140-143. 